\title{
Time-Resolved 3D Imaging of Ion Beam Induced Surface Damage in Gold Nanoparticles
}

\author{
Daniel Bufford ${ }^{1}$, Sarah H. Pratt ${ }^{2}$, Timothy J. Boyle ${ }^{3}$ and Khalid Hattar ${ }^{1}$ \\ ${ }^{1 .}$ Sandia National Laboratories, Radiation-Solid Interactions, Albuquerque, NM, USA \\ 2. Sandia National Laboratories, Analytical Technologies, Albuquerque, NM, USA \\ 3. Sandia National Laboratories, Advanced Materials Laboratory, Albuquerque, NM, USA
}

Many properties of nanoparticles (NPs) are closely tied to their size, shape, surface, and internal structures [1,2]. Different synthesis methods can produce an array of NP sizes and shapes, but it is also desirable to have methods to modify existing NPs. Extensive research on ion beam induced surface modification of bulk materials [3] has led to a strong understanding of ion-solid interactions, and positioned ion beams as promising tools for modifying NPs. However, fewer studies have explored the effects that these energetic ions have on nanomaterials [4,5]. Electron tomography is a useful tool for exploring the 3D shape of small structures, and can be applied to study ion beam induced structural modification in NPs. While general trends in NP shape evolution during ion bombardment can be obtained by ex situ irradiation followed by microscopy, such techniques cannot reliably track the evolution of individual particles. Additionally, diffusion may obscure subtle details of surface structures and sputtered material left immediately following an ion collision before it is possible to observe the specimen. In situ ion irradiation TEM addresses this issue by allowing nanometer-scale observation of samples in real time during bombardment by a variety of ion species and energies. By combining in situ irradiation and electron tomography techniques, samples can be continually monitored for changes, then immediately examined in greater depth when such changes occur.

In this work, we probe the effects of energetic heavy ion collisions on NP surface morphology and overall structure. Au NPs on holey carbon TEM grids were bombarded by $2.8 \mathrm{MeV} \mathrm{Au}{ }^{4}+$ ions, while in situ data were collected. Irradiation was stopped at select intervals, and serial tilt sections were collected, from which sequential tomograms were generated to reveal more detailed information about surface morphology changes produced in the NPs by ion bombardment (see Figure 1). Tomograms were computed using IMOD [6] and modified using UCSF Chimera [7].

A significant portion of incident ions caused only small changes in NP morphology, likely because they did not make direct collisions with atoms in the NPs. However, some more violent single ion collisions were directly observed, which resulted in sputtering of clusters or creation of bumps and nanoscale protrusions extending from the NP surfaces. Longer nanoscale protrusions either retracted back toward the surface, or separated into separate smaller particles. Figure 1 illustrates the development of roughened surfaces and sputtered particles resulting from several discrete collisions. Over time, the cumulative effects of repeated ion collisions caused sintering and agglomeration of initially closely spaced NPs. At the same time, sputtering processes produced a multitude of smaller satellite NPs $(\sim 1-2$ $\mathrm{nm}$ in diameter) surrounding parent NPs. During the most extensive periods of irradiation, it was observed that a significant amount of the initial volume of NPs was lost due to ablation by sputtering. This work provides insight into the processes involved in modifying the 3D surface structures of NPs, in addition to the potential utility of using ion beams to produce fine complex shaped NPs via sputtering [8]. 


\section{References}

[1] MC Daniel and D Astruc, Chemical Reviews 104 (2004), p.293-346.

[2] M Ferrari, Nature Reviews Cancer 5 (2005), p.161-171.

[3] O Auciello and R Kelly, "Ion Bombardment Modification of Surfaces: Fundamentals and Applications", (Elsevier Science Publishers, New York), p.1.

[4] AV Krasheninnikov and K Nordlund, Journal of Applied Physics 107 (2010), p.1-70.

[5] G Greaves, et al, Physical Review Letters 111 (2013), p.1-5.

[6] JR Kremer, et al, Journal of Structural Biology 116 (1996), p.71-76.

[7] EF Pettersen, et al, Journal of Computational Chemistry 25 (2004), p.1605-1612.

[8] Tomograms were modified using the UCSF Chimera package from the Computer Graphics Laboratory, University of California, San Francisco (supported by NIH P41 RR-01081). The authors thank S. House, K. Jungjohann, B.A. Hernadez-Sanchez, A.N. Kinghorn, J.S. Custer, and D.L. Buller for their helpful assistance. This work was supported by the Division of Materials Science and Engineering, Office of Basic Energy Sciences, U.S. Department of Energy. Sandia National Laboratories is a multi-program laboratory managed and operated by Sandia Corporation, a wholly owned subsidiary of Lockheed Martin Corporation, for the U.S. Department of Energy's National Nuclear Security Administration under contract DE-AC04-94AL85000.

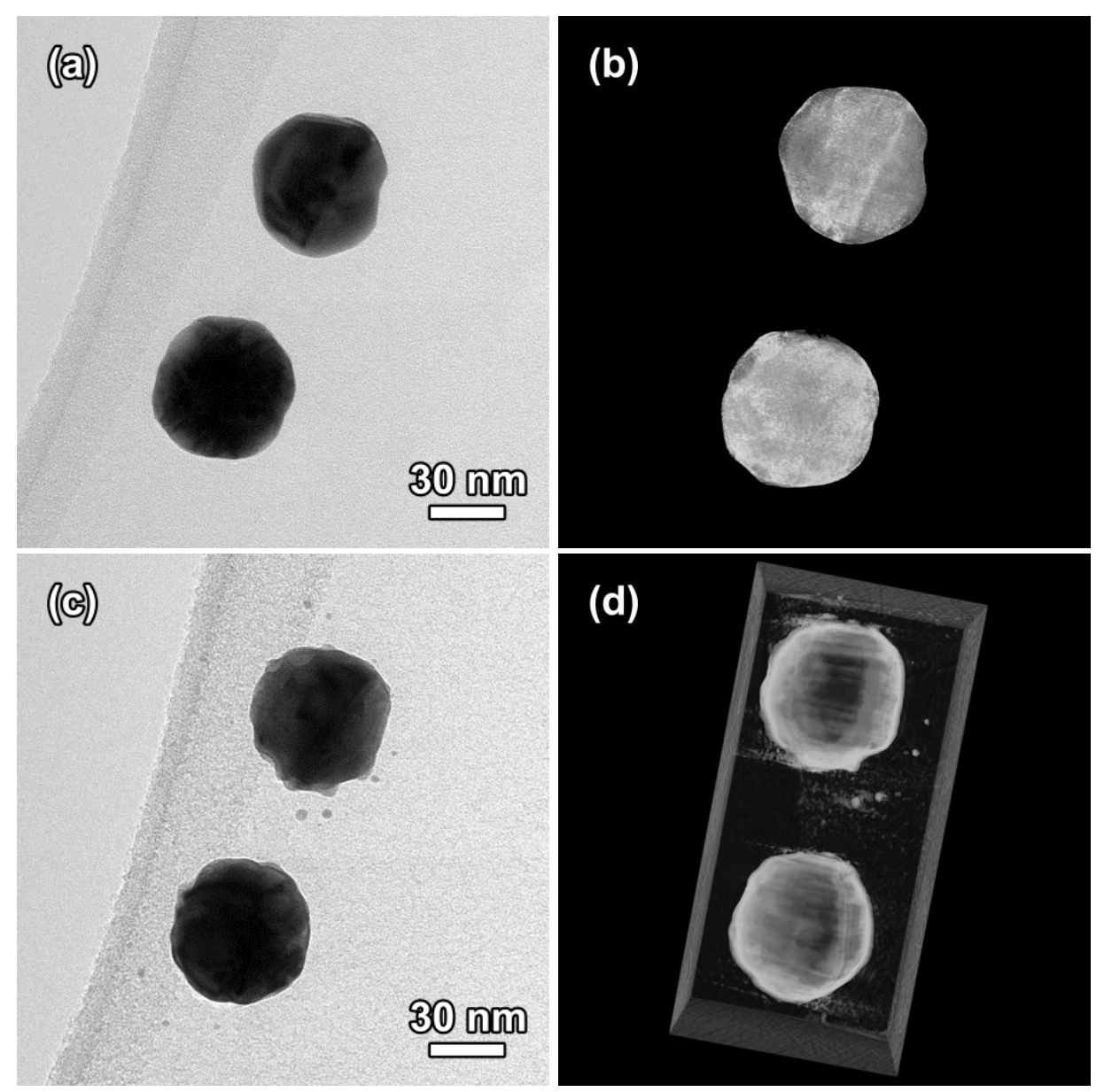

Figure 1. (a) Transmission electron micrograph and (b) tomogram showing pristine NPs, approximately $60 \mathrm{~nm}$ in diameter. (c) A second micrograph and (d) tomogram pair showing surface features and sputtered particles created by $2.8 \mathrm{MeV} \mathrm{Au}^{4+}$ irradiation to a fluence of $2.0 \times 10^{14}$ ions $/ \mathrm{cm}^{2}$. 